\title{
Balkanologie
}

Balkanologie Revue d'études pluridisciplinaires

Vol. III, n² | 1999

Volume III Numéro 2

\section{Combattants de la cause nationale : ethnicité et génocide dans les Balkans}

Ivelin Sardamov

\section{(2) OpenEdition}

\section{Journals}

Édition électronique

URL : https://journals.openedition.org/balkanologie/736

DOI : 10.4000/balkanologie.736

ISSN : 1965-0582

Éditeur

Association française d'études sur les Balkans (Afebalk)

Édition imprimée

Date de publication : 1 décembre 1999

ISSN : 1279-7952

\section{Référence électronique}

Ivelin Sardamov, "Combattants de la cause nationale : ethnicité et génocide dans les Balkans », Balkanologie [En ligne], Vol. III, $n^{\circ} 2$ | 1999, mis en ligne le 22 juin 2010, consulté le 28 juin 2022. URL http://journals.openedition.org/balkanologie/736 ; DOI : https://doi.org/10.4000/balkanologie.736

Ce document a été généré automatiquement le 17 décembre 2020.

(c) Tous droits réservés 


\title{
Combattants de la cause nationale : ethnicité et génocide dans les Balkans
}

\author{
Ivelin Sardamov
}

1 L'euphorie provoquée par la chute du mur de Berlin en 1989 s'est estompée, en même temps que la "fin de l'histoire", encore une fois annoncée, semble être indéfiniment reportée. La réapparition rapide des mouvements nationalistes et des conflits nationaux en Europe de l'est et ailleurs a symbolisé ce changement de perspective. Le "nouveau désordre" a fait l'objet d'un nombre croissant de travaux scientifiques sur le nationalisme et les conflits nationaux, certes variés et inégaux. La majeure partie des chercheurs s'intéressant à ce domaine partagent un important dénominateur commun. Ils ont adopté une approche "instrumentaliste": la récente crise de nationalisme ethnique et de violences intercommunautaires résulterait principalement de manipulations effectuées par des élites protégeant - ou tentant d'accéder à - des biens matériels, au pouvoir et au statut qui y sont liés ${ }^{1}$.

2 Le bon accueil qu'ont reçu ces théories "instrumentalistes" tient, au moins en partie, aux préoccupations politiques de la plupart des chercheurs tentant de "présenter" le nationalisme et les conflits nationaux de telle sorte qu'ils puissent paraître remédiables ${ }^{2}$. Ils ont, généralement, considéré la guerre et la violence intercommunautaires comme des "variables dépendantes" et tenté de découvrir leurs causes, afin de favoriser la prévention et la résolution des conflits. En quête de solutions réalisables, ils se sont souvent appuyés sur des analogies avec les sociétés occidentales, aux champs public, politique et économique hautement institutionnalisés. Ayant fixé l'agenda, nombre de chercheurs ont abouti à la conclusion que les conflits sont alimentés par les médias qui disséminent des identités collectives fictives et pernicieuses, et, par conséquent, que ce processus doit être réversible par le soutien à l'engineering politique. L'implication internationale dans la reconstruction des institutions démocratiques par l'organisation rapide d'élections et la transformation 
des identités nationales en identités "civiques" ou méta-nationales à la suite de conflits violents est alors sujet de grands espoirs.

3 Récemment, des chercheurs ont également tenté de renverser la tendance précédente qui dépeignait le monde non-occidental comme une région socialement "en retard" sous l'emprise de passions irrationnelles. D'autres ont été contraints de démontrer que, si l'opportunité leur était offerte, la plupart des individus de par le monde choisiraient de vivre librement dans des sociétés possédant une organisation sociale et humaine de type occidental. Ces deux groupes de chercheurs ont donc accusé les élites locales d'attiser les haines nationales alors qu'elles étaient (quasi) inexistantes, et de pousser des individus récalcitrants, se trouvant dans une situation précaire, à une violence intercommunautaire d'une grande ampleur. Bien que cette approche mette l'accent sur un aspect primordial de nombreux conflits nationaux, sa crédibilité est mise à mal par la tendance de ses adhérents à considérer cette explication comme exhaustive et à déconsidérer toute interprétation alternative.

4 S'appuyer sur des analogies avec des mécanismes sociaux, des attitudes publiques ou des pratiques d'engineering social de type occidental peut rapidement devenir problématique dans le cadre de la composition sociale des Balkans, plus volatile et encore très largement corporatiste. En dépit d'avancées dans l'industrialisation, l'urbanisation et l'éducation, les structures parentales, les traditions orales et les mécanismes informels de relations sociales sont toujours partie intégrante du tissu social dans la région. Leur élasticité pose un énorme obstacle à la propagation, par les médias ou les organismes non gouvernementaux, des valeurs individuelles et universelles occidentales. La survivance de structures sociales et de formes de relations sociales "traditionnelles" n'est pas seulement la résultante du sous-développement économique et social. Elle a également été renforcée par la longue histoire des invasions et dominations étrangères, par les luttes pour l'autodétermination et le partage des possessions des empires défunts. Par conséquent, notre compréhension des dynamiques des nationalismes et des conflits nationaux dans les Balkans doit s'appuyer sur un examen minutieux de la signification qu'ont les luttes anciennes pour l'indépendance nationale et l'"unification" pour les identités nationales conflictuelles des belligérants.

\section{Le poids de l'histoire?}

5 Un examen du "poids de l'histoire" dans la région montre que les conflits passés et les ressentiments nationaux, certains vieux de plusieurs siècles, ont conservé un haut degré de pertinence dans les débats politiques contemporains. C'est un phénomène qui ne peut pas s'expliquer par les seules habiletés manipulatrices et l'imagination débridée des politiciens et intellectuels locaux. Ce qui est commun en Europe de l'est, et que G. Steiner a appelé la «nécessaire tension du passé " d'une "grammaire [communautaire] de l'être $\aleph^{3}$, est particulièrement vive parmi les populations balkaniques. Et ce n'est pas une surprise s'il existe une parcelle de vérité dans l'assertion de C. Milosz que la conscience aiguë de l'histoire en Europe de l'est est le résultat d'une menace mortelle perçue par chaque communauté nationale ${ }^{4}$. À quelques exceptions près, les peuples balkaniques ont vécu pendant des siècles sous domination étrangère, surtout en tant que paysans confinés au bas de l'échelle sociale aussi bien dans l'Empire ottoman que dans l'Empire habsbourgeois. Ils ont ensuite été incorporés 
dans des États nationaux ou plurinationaux, et ont été pris dans les longues et violentes luttes de pouvoir régionales.

6 La pertinence des références à des événements ou des personnages de l'histoire ancienne, dans la région, provient également des modes narratifs et imaginaires véhiculés par les cultures populaires locales qui ont, à une très large échelle, évolué en dehors des canaux officiels. Bien qu'elles aient été significativement influencées par la haute culture et l'endoctrinement nationaliste, elles ont précédé la littérature moderne ainsi que les traditions intellectuelles de la région et $\mathrm{y}$ ont apposé une marque indélébile ${ }^{5}$. Le folklore, la littérature et l'histoire interprètent souvent de manière plutôt similaire la structuration sociale, l'organisation politique et les motivations humaines.

7 La récente mobilisation des ressources nationales dans une partie des Balkans peut être analysée comme partie intégrante d'une longue suite événementielle, plutôt qu'un phénomène contemporain unique. Depuis le siècle dernier, les leaders nationalistes locaux n'ont jamais cherché à obtenir un large soutien populaire sur leurs promesses tendant à défendre les intérêts particuliers de leurs électeurs. Ils se sont régulièrement référencés aux événements et personnages du passé pour décrire les groupes nationaux voisins comme des ennemis historiques afin de légitimer et de conserver le pouvoir politique. Ils ont également évoqué des impératifs trans-générationnels, présentant les États nationaux nouvellement établis comme des réincarnations de glorieux royaumes médiévaux et les contestations politiques et militaires comme la reproduction de combats historiques : une nouvelle bataille pour Kosovo Polje, la réaffirmation du droit à l'État indépendant du peuple croate, l'insistance sur les torts causés au peuple bulgare à la suite du traité de San Stefano, etc. Même lorsque de telles références historiques sont employées comme un camouflage cynique d'objectifs politiques plus pragmatiques, le fait qu'elles aient souvent été effectives pour susciter une réponse massive d'individus normalement sceptiques est significatif en soi.

8 Afin d'attirer un large soutien populaire dans leurs luttes pour le pouvoir, la légitimité et une place dans l'histoire, les élites balkaniques ont dû favoriser le développement d'institutions et de doctrines nationales qui ne sont qu'imparfaitement instrumentales, aux objectifs et aux fonctions strictement délimités. Elles ont promu des institutions et des "idéaux" nationaux qui peuvent être perçus non seulement comme des moyens pratiques de promotion sociale, mais également comme le fondement de toutes les aspirations nationales et la garantie d'une identité commune rédemptrice. C'est le flou de ce mandat existentiel qui lui permet d'être souvent utilisé, notamment dans les périodes d'effondrement des empires et d'agitation internationale. Le mandat en luimême a, de loin, surpassé les messages explicites de la propagande nationaliste moderne et a fourni certains standards pour l'évaluation de la "qualité" des leaders nationalistes fanatiques ou cyniques.

9 Le "traditionalisme" balkanique est également lié à une autre particularité qui explique en grande partie le lourd fardeau de l'histoire dans la région. Il a contribué au développement d'un certain degré d'ethnocentrisme populaire mettant en exergue l'ascendance ou le sang communs comme base naturelle de la communauté politique, et a empêché ainsi la formation de nations méta-nationales sur le modèle occidental ${ }^{6}$. Sans aucun doute, cet ethnocentrisme exclusif, et parfois violent, a été promu par des leaders et des intellectuels nationalistes pour leurs propres finalités politiques. Leurs efforts dans l'endoctrinement nationaliste ont été facilités par la propagation de 
nouvelles notions d'unité sociale liées à des innovations historiques majeures: l'apparition de la sphère publique en tant qu'espace commun de dialogue sur les mœurs et le gouvernement, ainsi que la généralisation de l'éducation sécularisée; le développement de structures politiques centralisées étendant leur contrôle administratif sur des populations plus nombreuses et dont la légitimité dépend de la sphère publique; et la transformation d'une économie reposant sur une structure essentiellement familiale à un système de flux massifs de biens, de services et d'instruments financiers aux impératifs spécifiques? ${ }^{7}$.

10 Toutefois, le contrôle des actions et des opinions de leurs populations, ainsi que la demande de services exigés par les États balkaniques contemporains, ou ce que Michael Mann appelle le "pouvoir infrastructurel" de l'État ${ }^{8}$, ne doivent pas être surestimés. En dépit des ambitions des leaders souvent autoritaires et de leur entourage, les appareils d'État balkanique n'ont que rarement disposé des ressources administratives nécessaires et de l'aptitude à pénétrer tous les niveaux de la société afin de supprimer les racines des réseaux et les solidarités sociales ou d'établir un contrôle social efficient $^{9}$. Ceci est vrai y compris pour les régimes communistes de Yougoslavie, Bulgarie, et Albanie, malgré le label "totalitaire" qui leur a été parfois attribué. Les identités nationales balkaniques actuelles ne proviennent donc pas seulement des messages de la propagande nationaliste institutionnalisée, mais également de la tendance générale des individus au sein de sociétés "traditionnelles" à s'identifier et à être identifiés par les autres surtout en fonction de leur appartenance à des groupes sociaux. Dans les Balkans, de tels modèles d'identification et de classification ont persisté pendant des siècles, bien que le groupe auquel les individus s'identifient se soit déplacé de la communauté locale et religieuse à la communauté ethnoreligieuse et, parfois, nationale.

11 Les approches ethnocentriques de l'identité nationale ont rendu pertinent le recours aux références historiques, en liant les expériences des générations passées et actuelles d'un même ethnos commun à un tout indifférencié. Selon l'historien bulgare Roumen Daskalov:

Notwithstanding demographic catastrophes and ethnic creolage, even if these are admitted, "we" living today are identical with the historical "them", our forefathers. They are the earlier "we", we are the later "them" - that is what is conveyed and implied by the [historical] narrative. There are no limits to the projection of ethnicity back into history; since the tribal period history is "our" history. ${ }^{10}$

12 Les intellectuels nationalistes des États plurinationaux ont également tenté de réaliser une telle fusion de générations distantes en un seul protagoniste d'un drame historique intemporel, mais leur tâche fut bien plus ardue et ils n'ont que rarement réussi.

Des exemples de définition ethnocentrique commune présentant les groupes nationaux situés sur un espace comme des espèces naturelles aux caractéristiques innées indélébiles sont faciles à trouver ${ }^{11}$. Dans l'ancienne Yougoslavie, de telles notions ont été au cœur de débats animés affirmant que le narod (ethnos) est plus pur et racialement supérieur et que "les Croates" sont marqués d'une "nature génocidaire" commune. Plus sérieusement, la définition précise de l'appartenance nationale a sous-tendu les revendications serbes d'une Yougoslavie produit d'une union des peuples plutôt que des États pré-existants. Ainsi, les peuples constitutifs jouissaient du droit à l'autodétermination, et non pas les Républiques, unités territoriales et administratives. $\mathrm{Au}$ contraire, la République de Croatie a été clairement constituée sur une base 
nationale, l'État du - et pour le - peuple croate. Le Parlement croate, élu en 1990, a accordé un droit de vote automatique aux centaines de milliers d'expatriés croates, dont la minorité croate de la République de Bosnie-Herzégovine souveraine. L'État bulgare a également existé pendant plus d'un siècle comme l'État national du narod bulgare. La culture et la politique bulgares sont encore marquées par l'argumentaire courant affirmant que "les Macédoniens" d'aujourd'hui sont en fait des Bulgares, d'autant plus que la constitution bulgare de 1991 reconnaît comme citoyen bulgare toutes les personnes dont les parents sont d'origine nationale bulgare ${ }^{12}$. Les présidents de Macédoine et d'Albanie ont récemment échangé des propos caractéristiques: critiquant Kiro Gligorov qui avait déclaré que les réfugiés albanais devraient aller dans leur patrie, l'Albanie, Rexhep Meidani a rétorqué que les Albanais de la région vivaient sur leur propre "territoire national"13. L'empressement récent de la population albanaise à la frontière yougoslave à aider les nombreux réfugiés du Kosovo démontre clairement le degré que les sentiments de "fraternité" ou de parenté nationales naturelles peuvent atteindre dans la région.

\section{Communautés « historiquement » ennemies}

Bien que les conceptions ethnocentriques de l'identité conduisent à une compassion nationale spontanée de la population albanaise, elles ont également de graves conséquences. Tout d'abord, elles impliquent une prédisposition à reconnaittre les acteurs collectifs, et non pas individuels, comme éléments constitutifs des communautés politiques et les véritables détenteurs des droits, des devoirs, et même de la culpabilité. Les caractéristiques des communautés nationales voisines, transformées en ennemis mortels collectifs par le choc des missions "nationalisantes", ainsi que celles des individus les représentant, sont considérées comme innées et, par conséquent, irréversibles. Ils sont exclus de ce qu'Andrei Simić décrit comme «un champ moral [commun], un champ interactionnel dans lequel chacun agit envers l'autre par référence à des impératifs perçus comme nationaux $»^{14}$. De tels individus ont souvent été les sujets de mauvais traitements sur la seule base de leur origine nationale, d'assimilation forcée, parfois même de violentes campagnes de "nettoyage ethnique". Ensuite, les membres des minorités nationales sont systématiquement réduits au statut de citoyens de deuxième classe, "invités" dans l'État d'un autre groupe national, quelles que soient les garanties constitutionnelles ou internationales de leurs droits ${ }^{15}$. Enfin, la tendance à considérer la langue et les traits culturels comme les indicateurs d'une ethnicité commune a abouti à douter de l'authenticité de l'autoidentification d'autres groupes nationaux, et à des conflits perpétuels à propos de l'identité "réelle" de groupes plus petits, comme les Musulmans bosniaques ou les Macédoniens aujourd'hui.

15 Dans ces conditions sociales, les luttes historiques des peuples balkaniques ont révélé un paradoxe. D'un côté, elles ont parfois inspiré compassion, persévérance et de nombreux sacrifices. De l'autre, elles ont semblé dresser l'une contre l'autre des populations entières, plutôt que des armées régulières, dans des campagnes de génocide. Elles ont donc occasionné un haut degré de violence intercommunautaire : le massacre de civils ou de prisonniers de guerre sur la seule base de leur origine nationale par des unités irrégulières, et parfois régulières, l'incendie de villes et villages, ainsi que le déplacement de populations, ce qui sera appelé "nettoyage ethnique" ${ }^{16}$. La justification de ces actes, aussi bien que le besoin primordial d'auto- 
sacrifice $^{17}$, se trouve dans l'évocation de la mémoire de la gloire et des souffrances anciennes liées aux conflits nationaux antérieurs.

Pendant longtemps, des tendances génocidaires ont marqué les guerres, les rébellions et les conflits intercommunautaires dans les Balkans sud slaves. Mais, soutenir que l'histoire de la région a été gelée et qu'elle est condamnée à se répéter dans ses conflits nationaux "barbares" est une simplification à outrance ${ }^{18}$. Tandis que l'autoidentification ethnocentrique de majorités au sein des populations locales est plutôt identique à celle de leurs ancêtres du siècle dernier, voire des siècles antérieurs, les guerres et les conflits nationaux contemporains ont été marqués par une modification radicale. Ce changement est principalement lié à la structure institutionnelle de la guerre. Jusqu'à la fin du XIX ${ }^{\text {ème }}$ siècle, ce sont essentiellement des troupes autonomes irrégulières qui s'affrontaient. Les individus qui y participaient disposaient souvent d'un sens aigu de l'appartenance "naturelle" à leur communauté ethnoreligieuse et, en même temps, d'un fort sentiment d'hostilité envers les autres personnes. La distinction entre combattants et non-combattants était plutôt floue et tous les membres des communautés rivales étaient perçus comme des cibles légitimes. Les détachements armés comprenaient habituellement des combattants implacables et brutaux, mais les structures et valeurs sociales traditionnelles imposaient des limites importantes de degré et d'intensité dans les hostilités. Les bandes armées, souvent de petite taille et très mobiles, n'opéraient pas à partir d'une base permanente et disposaient d'armes et d'une logistique plutôt primitives. Bien que les atrocités régulières qu'elle commettaient sur les non-combattants étaient parfois réalisées à grande échelle, la violence était, le plus souvent, en partie ritualisée et, sauf de rares occurrences, ne s'orientait pas vers une "solution finale" 19 .

Les choses n'ont dramatiquement changé qu'avec l'incorporation de nombreux soldats croyant aux notions d'attachement ethnique organique et irrévocable dans les armées modernes disposant d'une puissance de feu destructrice et de moyens logistiques accrus. Ces armées, composées de "soldats nationaux" traditionalistes, étaient au service d' "États se nationalisant " $^{20}$ en quête d'expansion territoriale, à l'époque où l'effondrement des empires multinationaux promettait la création de patries nationales indépendantes qui, idéalement, uniraient tous les membres du même ethnos et permettraient aux co-nationaux présumés de retrouver leur "vraie" identité. Cette perspective a facilité la violente rencontre des missions nationalisantes et la perception accrue des autres communautés nationales comme des ennemis historiques marqués par des caractéristiques innées opposées. Ces tendances ont alimenté de nombreuses guerres et conflits nationaux sanglants qui ont été bien plus destructeurs que nombre de conflits du siècle dernier : les guerres balkaniques de 1912-1913, les combats internationaux en Yougoslavie au cours de la Deuxième Guerre mondiale, et les conflits marquant l'éclatement de la Yougoslavie dans les années 1990 (y compris la guerre au Kosovo en 1999). Tous ces conflits présentent bien plus d'aspects génocidaires que ceux s'étant déroulé au sein des Empires multinationaux.

\section{Utilisations du folklore}

18 Dans les Balkans, les conflits nationaux ont longtemps été commémorés en tant qu'éléments de traditions nationales vénérables. Les récits sélectionnés et dramatisés à cet effet jouent un rôle central dans les histoires nationales balkaniques. Ils obéissent à des schémas conceptuels hautement standardisés. Bien que ces récits présentent un 
fort aspect "mythique", ils se réfèrent également à des événements historiques "réels" qui ont eu d'importantes conséquences sur la vie de millions de personnes. Invariablement, ils mettent l'accent sur les souffrances prolongées subies sous la coupe d'oppresseurs étrangers et sur les soulèvements populaires pour la "libération" nationale et "l'unification", liant les expériences des générations passées et présentes en un tout cohérent. Les événements et personnages historiques majeurs sont transformés pour servir les buts des leaders et intellectuels nationalistes. Ces derniers, toutefois, ont souvent défini leurs propres objectifs politiques en interprétant de façon rigide quelques exemples issus de l'histoire. Ces interprétations ont évolué. Elles sont le résultat des efforts de générations d'écrivains, d'historiens et d'autres intellectuels nationalistes. Elles ont également suivi les conventions et les analyses informelles des classifications, causalités et motivations sociales présentes dans les récits de tradition orale et dans les relations sociales. En dépit de tensions internes, elles ont nourri les identités nationales balkaniques d'aujourd'hui avec un degré d'unité et de cohésion bien éloigné des processus de fragmentation culturelle de la plupart des sociétés occidentales.

Dans une série d'analyses brillantes, Ivan Čolović, l'un des intellectuels libéraux serbes des années récentes les plus respectés, a démontré l'efficacité des formules folkloriques dans la fédération yougoslave, et notamment en Serbie ${ }^{21}$. Il a porté son attention sur les commentaires journalistiques qui, dans les années 1980, ont présenté les matchs de football et les footballeurs yougoslaves comme des reconstitutions de batailles mythiques paradigmatiques avec un ennemi déshumanisé et ont interprété le style de jeu de chaque équipe comme une "expression de la mentalité nationale". Il a étudié des rumeurs et des écrits qui ont fait d'un criminel serbe, assassiné dans les années 1980 en Allemagne, un héros populaire: un noble protecteur des défavorisés, même s'il a parfois été cruel, dans la plus pure tradition des hajduks. Il s'est également intéressé aux "nouvelles chansons populaires" et aux slogans présentant Milošević comme une nouvelle incarnation d'un héros aux dimensions quasi-mythiques, le "père-roi" serbe $\mathrm{du}$ folklore épique (bizarrement, Vuk Drašković a été dépeint en termes identiques dans les chansons du $\mathrm{SPO}^{22}$ ), ainsi qu'à de nombreuses autres chansons de style similaire décrivant la guerre yougoslave récente comme une continuation de la tradition serbe tant vénérée des luttes de "libération", mêlant événements et personnages historiques sans liens entre eux. Selon Čolović, ces genres populaires de "littérature sauvage" ont "leur origine dans la littérature générale et le développement des moyens de communication, qui ont modifié, en grande partie, la forme de notre culture, mais non son esprit $»^{23}$.

Lors des nombreuses guerres balkaniques, les rébellions et les exploits des chefs hajduk ont longtemps été commémorés avec une dévotion particulière. La vénération pour les actions "héroïques" de l'un des "ancêtres" du groupe national a été systématiquement inculquée au travers du système scolaire, du service militaire obligatoire, de la propagande écrite et, depuis peu, visuelle. Ces efforts ont trouvé un terrain fertile, défriché par la tradition orale. Cette particularité est surtout évidente dans le cas du folklore serbe et des relations sociales qui ont véhiculé des contes épiques détaillés des luttes passées, jusqu'à la célèbre bataille de Kosovo Polje en 1389. Le contenu quasihistorique d'autres traditions folkoriques dans la région était bien moins prononcé. Elles ont, généralement, affirmé et insisté sur la division naturelle du monde en ethnes ethniquement définies, l'identité des différentes générations appartenant au même 
ethnos et l'unité essentielle du passé avec le présent ${ }^{24}$. Elles ont ainsi fourni un cadre interprétatif approprié aux allusions historiques récurrentes de nombreux écrivains, historiens et politiciens.

Des intellectuels et des politiciens locaux ont peut-être suivi les conventions du folklore local et des relations sociales relatives à leur origine rurale ou semi-rurale. D'autres, toutefois, ont trouvé des formules folkloriques suffisamment évocatrices pour la majorité de leur public ou de leur électorat co-national. Le plus souvent, les Milošević et autres Tuđman ont atteint les sommets du pouvoir en exploitant la vague nationaliste dont ils n'étaient pas les seuls initiateurs et qui, s'ils n'en avaient pas profité, aurait servi à d'autres leaders nationaux. Ils détiennent des positions d'autorité en vertu d'un "mandat de l'histoire" englobant des pratiques particulières d'action politique légitime. Bien qu'ayant très bien réussi à manipuler quelques aspects de ce mandat, la portée de leurs efforts a été restreinte par son dynamisme général.

Présenter les contestations politiques et militaires actuelles dans les Balkans comme des reconstitutions des luttes passées a souvent été un moyen politique efficace dans les mains des élites politiques. Mais, c'est parfois devenu une arme à double tranchant. L'utilisation de références historiques a permis aux leaders politiques et aux intellectuels d'obtenir un large soutien populaire. Ils ont été entraînés dans des rôles historiques qu'ils n'étaient que partiellement prêts à interpréter. De telles dynamiques les ont rendus vulnérables au ressentiment populaire lorsqu'ils n'arrivent pas à tenir les promesses qu'ils ont faites. Quand les leaders nationalistes échouent à remplir le "mandat historique", ils perdent beaucoup de la légitimité nécessaire à leur revendication de pouvoir et de direction. C'est ce qui est arrivé à Ante Pavelić et son régime après qu'ils aient proclamé le $\mathrm{NDH}^{25}$ réincarnation du royaume croate, alors qu'ils furent contraints d'abandonner une bonne partie du territoire à l'Italie et de faire du NDH un protectorat de l'Axe. Dans ce contexte, l'intransigeance de Milošević face à l'ultimatum de l'OTAN et des bombardements reflète probablement une conscience aiguë de la charge de leader national serbe qu'il a dû assumer pour rester au pouvoir.

Quand les défaites militaires sont particulièrement douloureuses et humiliantes, elles portent un coup à la légitimité des élites politiques et aux institutions qu'elles contrôlent. Au lendemain de la Première Guerre mondiale, par exemple, l'État bulgare s'est aliéné les masses paysannes et une bonne partie de l'intelligentsia. En dépit de l'endoctrinement nationaliste par les canaux officiels, elles ne pouvaient plus être mobilisées pour soutenir les institutions et la politique étatiques, dont la "rédemption" des territoires perdus après le traité de San Stefano. Il reste à voir si des processus similaires se développeront en Serbie après la campagne aérienne de l'OTAN.

\section{Transcender l'identité}

24 Une des particularités de l'auto-identification ethnique balkanique, parfois relevée par des observateurs étrangers, réside dans sa dimension quasi-transcendentale, qui n'a que peu de rapport avec la religion. Du fait des diverses traditions religieuses, un " folklore religieux, localisé et basé sur la superstition » a vu le jour ; il a surtout servi de symbole d'identité collective et de différenciation par rapport aux autres communautés ${ }^{26}$ ou de "totem clanique ${ }^{27}$. Comme Anthony Smith et Benedict Anderson ont tenté de le démontrer, ce qui distingue l'identité nationale des autres formes d'identité sociale est sa capacité à conférer aux individus l'impression d'une 
immortalité terrestre. Elle y parvient en faisant des individus le lien collectif d'une chaîne ininterrompue de générations appartenant à une "communauté territoriale et culturelle" plus large $\mathrm{e}^{28}$. Cet aspect transgénérationnel est notamment prononcé dans le modèle ethnique de la nation, avec son insistance sur l'ascendance physique et la révérence au passé ethnique. En temps de crise et d'insécurité, il peut détourner les individus de leur quotidienneté et les faire adopter ce qu'ils considéreraient autrement être de la marginalité. Une telle quête désespérée de transcendance par la participation à un acte de rédemption communautaire est probablement la principale raison de la motivation qui a poussé la masse des "soldats-libérateurs" nationaux dans les Balkans sud slaves à répondre aux appels à prendre les armes qui se sont succédés ces dernières décennies. Bien que certains d'entre eux soient des criminels de droit commun, tel le célèbre Arkan, ou des mégalomaniaques obsédés, tels Vojislav Šešelj ou Dobroslav Paraga, la plupart ont cherché plus que le butin de guerre, la réalisation de désirs sadiques, ou ont été intoxiqués par le pouvoir sur la vie qui leur été conféré. Beaucoup ont participé à des guerres pour la survie physique ou la rédemption de leur ethnos qui exigeait des efforts, parfois même des sacrifices, ainsi que de la brutalité contre les membres des communautés "ennemies".

L'approche "instrumentaliste" maintenant prédominante dans l'étude du nationalisme et des conflits ethniques, soulignant les manipulations par des élites affamées de pouvoir sur des masses dociles, met en exergue des aspects importants de nombreux conflits ethniques, mais elle ne peut pas complètement rendre compte de leur férocité et de leur cruauté excessives. Elle généralise bien trop rapidement les expériences et interprétations propres aux sociétés occidentales hautement institutionnalisées et individualistes. La plupart d'entre elles sont organisées selon des clivages supranationaux, dans lesquels les cultures folkloriques ont été remplacées par les médias, eux-mêmes contrôlés par les sphères publiques. Selon l'expression du critique audiovisuel, Robert Hughes, la télévision est devenue le "principal générateur de la réalité $»^{29}$. Les tenants de l'approche "instrumentaliste" tendent à extrapoler ces expériences et ces interprétations sans porter de regard critique sur les régions dont les caractéristiques sociales, culturelles et politiques sont différentes. Afin de mieux appréhender la dynamique des rivalités nationales dans les Balkans, il est également nécessaire de prendre en considération le pouvoir de la mémoire des guerres passées pour fournir des normes pratiques d'action politique légitime.

Malheureusement, ces normes ont trop souvent sanctionné une résolution violente des conflits et des atrocités contre les masses "ennemies" non-combattantes. De tels outrages, toutefois, ne peuvent pas être expliqués par les seules cruauté, quête du pouvoir et capacités manipulatrices d'un petit nombre de politiciens et d'intellectuels nationalistes. Des excès aussi tragiques ont accompagné la naissance de pratiquement tous les mouvements sociaux majeurs de l'histoire de l'Europe moderne : de la Réforme aux guerres du XXème siècle, en passant par la Révolution française. Bien que ce fait soit négligé, même les démocraties occidentales actuelles se sont étendues et consolidées au travers de siècles de guerre ; elles ont, elles aussi, procédé à la nationalisation de leurs populations par des politiques assimilationnistes ; elles ont mené des régimes coloniaux durs de par le monde.

27 Tous ces cataclysmes sociaux montrent peut-être la prédisposition humaine à la violence, une assertion suprême de l'identité collective au coût d'une possible destruction mutuelle ${ }^{30}$. On peut analyser cette tendance comme le problème le plus 
dérangeant de l'humanité depuis des temps immémoriaux, un problème que la plupart des sociologues et politologues a esquivé. Ils ont tenté d'exonérer les masses d'individus ordinaires des sociétés contemporaines en faisant porter le blâme de l'intolérance et de la violence intercommunautaire sur quelques "entrepreneurs" politiques en quête de pouvoir et d'un meilleur statut. La manipulation par des politiciens impitoyables et des intellectuels pathétiques est sans aucun doute un facteur important dans l'éclatement de nombreux conflits. Mais elle ne fournit aucune explication quant à leurs racines et leur dynamique. Faire de millions d'individus des robots dépourvus de tout esprit critique pouvant être facilement dupés et assumer des identités fictives, sacrifier leurs vies et celles des autres pour satisfaire un petit groupe de manipulateurs doués est une analyse simpliste et condescendante ${ }^{31}$. Si l'intention est louable, elle ne fait pas grand chose pour promouvoir les valeurs et institutions démocratiques que ses initiateurs cherchent pourtant à encourager. Il faut du temps et des moyens pour légitimer une nouvelle forme d'habitus culturel.

\section{Instrumentalisme et primordialisme}

Les références à des événements et des personnages historiques dans les Balkans ont parfois produit le résultat escompté, et parfois non. Les leaders et les intellectuels nationalistes ont parfois étendu leur popularité en se présentant comme les champions d'une tradition nationale vénérable; ils ont parfois été contraints d'imiter leurs prédécesseurs "héroïques". Quoiqu'il en soit, que les populations locales aient été incitées à l'action sur la base des luttes collectives, des souffrances et des injustices passées (datant parfois de plusieurs siècles) ou non, de telles références ont, le plus souvent, eu pour la majorité des accents de vérité. Il s'agit d'un phénomène oublié depuis longtemps dans les sociétés occidentales modernes ${ }^{32}$, dont l'éloignement $d u$ passé lointain ne peut pas être attribué aux tactiques différentes des politiciens et des intellectuels occidentaux. Comprendre la pertinence plus ancrée des références ethnohistoriques dans les luttes politiques actuelles représente une tâche indispensable si nous voulons comprendre la dynamique des rivalités nationales actuelles dans les Balkans.

29 La critique présente des théories "instrumentalistes" n'implique pas que les groupes ethniques ou les nations balkaniques doivent être analysés comme étant naturels, une association humaine éternelle, selon la "tradition primordialiste". Si les études récentes ont établi quelque chose, c'est que de tels groupes ne sont pas des communautés primales représentant des extensions directes de liens de parenté, reliant les individus dans le temps et l'espace sur le fondement d'un "sang", d'un territoire, ou d'autres éléments humains communs. Les nations et les groupes ethniques doivent, sans aucun doute, être considérés comme des entités historiques ayant émergé à partir des expériences, des pensées et des actions individuelles et ayant subi de larges transformations. Dans les Balkans, toutefois, ces processus se sont largement révélés être un phénomène populaire ${ }^{33}$. Une partie essentielle de leur dynamique a été dessinée à la base et a surpassé les efforts manipulatoires et la propagande des politiciens et des intellectuels nationalistes. L'identité nationale et l'ethnicité ont formé une partie d'un horizon existentiel qui, pour des millions d'individus, a non seulement inclus la distribution des fonctions et des rares ressources, mais également fourni un début de réponse aux questions relatives au sens de la vie, de la mort et de l'oubli. 

"primordialisme des participants", à savoir un sentiment de partager des liens ethniques originaires irrésistibles avec les membres d'une même communauté ethnique $^{34}$. De tels sentiments représentent «des données vitales pour enquêter sur l'ethnicité » et ne doivent pas être rejetés pour leur manque d'adéquation avec les interprétations en termes "primordialistes" ${ }^{35}$. Ils doivent être sérieusement étudiés par les tenants du "constructivisme" ou de "l'instrumentalisme" qui proclament que les identités et les liens sociaux sont ce que les individus en font, en tant qu'agents sociaux. Porter l'attention exclusivement sur les capacités manipulatrices de leaders peu scrupuleux, aussi important cela soit-il, peut être conceptuellement élégant, moralement commode et politiquement opératoire. Il semble toutefois qu'une approche intellectuellement problématique puisse aboutir à des diagnostiques, des pronostics et des prescriptions politiques contestables.

Sur le territoire de l'ancienne Yougoslavie, le "primordialisme des participants" qui a prévalu parmi les membres des différents groupes nationaux a été considérablement renforcé par le déploiement de la violence. À court terme, la primauté des conceptions ethnocentriques de l'identité nationale pourrait peut-être être atténuée par quelques changements dans le système éducatif. Afin d'être effectives, ces modifications ne doivent pas mettre l'accent sur l'inadéquation, ni le manque d'authenticité des identifications ethniques, mais plutôt se concentrer sur une présentation mitigée des luttes de "libération" des ethnes voisines. Une telle présentation démontrerait qu'aucune des ethnes de la région n'a le monopole sur la souffrance et le droit à une "rédemption historique". Des changements plus profonds des identifications et classifications sociales ne peuvent que difficilement avoir lieu par la seule action de quelques organisations non gouvernementales locales et internationales, le maintien de quelques sources d'informations indépendantes et des pressions politiques, ou même militaires, des gouvernements occidentaux. Ils ne peuvent résulter que de processus sociaux complexes qui risquent fort d'être douloureux et de prendre un certain temps à se réaliser. Dans les mois à venir, une défaite serbe percutante pourrait probablement tailler les ailes du nationalisme serbe. Le problème est que le coût humain en serait très élevé et la société serbe souffrirait probablement durant des décennies de la détérioration de la vie publique que la démobilisation sociale et le cynisme excessif généralisé peuvent attirer - un sort trop bien connu par la société bulgare depuis la fin des années 1920.

\section{NOTES}

1. Voir Väyrynen (Raimo), Towards a Theory of Ethnic Conflicts and Their Resolution, Occasional Paper \#6 (Notre Dame: Joan B. Kroc Institute for International Peace Studies, 1994) ; Ryan (Stephen), Ethnic Conflict and International Relations, Aldershot (UK) : Dartmouth, 1995 (2 ${ }^{\text {nd }}$ ed.).

2. Ce lien m'a été suggéré par Martha Merritt, un membre inestimable de mon comité de discussion à the University of Notre Dame.

Balkanologie, Vol. III, n² | 1999 
3. Steiner (George), In Bluebeard's Castle : Some Notes Towards the Redifinition of Culture, New Haven : Yale University Press, 1971, p. 3.

4. Milosz (Czeslaw), "About Our Europe ", in Kostrzewa (Robert), ed., Between East and West: Writings from Kultura, New York : Hill and Wang, 1990, pp. 100-101.

5. Voir Lord (Albert B.), « Nationalism and the Muses in Balkan Slavic Literature in the Modern Period ", in Jelavich (Charles), Jelavich (Barbara), eds., The Balkans in Transition: Essays on the Development of Balkan Life and Politics since the Eighteenth Century, Berkeley : University of California Press, 1963; Halpern (Joel Martin), Hammel (Eugene A.), «Observations on the Intellectual History of Ethnology and Other Social Sciences in Yugoslavia ", Comparative Studies in Society and History, 11 (1), 1969.

6. Cf. Just (Roger), "Triumph of the Ethnos", in Tonkin (Elizabeth), McDonald (Maryon), Chapman (Malcolm), eds., History and Ethnicity, London : Routledge, 1989.

7. La présentation de cet élément doit beaucoup à la contribution de Charles Taylor à the University of Notre Dame en avril 1992.

8. Mann (Michael), The Sources of Social Power,vol. 1, Cambridge : Cambridge University Press, 1986, p. 477.

9. Rebecca West médita sur la nature anarchique "du Slave" qui, selon elle, l'empêchait d'être le sujet de quelque pouvoir politique que ce soit. Cf. West (Rebecca), Black Lamb and Grey Falcon: A Journey Through Yugoslavia, New York : Viking Press, 1941, p. 306.

10. Daskalov (Roumen), Building up a National Identity : The Case of Bulgaria, working paper SPS no. 94/11 (Florence : European University Institute, Department of Political and Social Sciences, 1994), p. 4.

11. Voir Hall (Brian), The Impossible Country: A Journey through the Last Days of Yugoslavia, Boston : David R. Godine, 1994, p. 139.

12. Konstitutsiia na Republika Bulgaria [Constitution de la République de Bulgarie], Sofia: Sofia Press Agency, 1991, art. 25.

13. Cf. RFE/RL Newsline, 09/04/99.

14. Voir Simić (Andrei), «Obstacles to the Development of a Yugoslav National Consciousness : Ethnic Identity and Folk Culture in the Balkans ", Journal of Mediterranean Studies, 1 (1), 1991, p. 31. 15. Voir Hayden (Robert M.), "Constitutional Nationalism in the Formerly Yugoslav Republics ", Slavic Review, 51 (4), 1992.

16. Cf. The Other Balkan Wars: A 1913 Carnegie Endowment Inquiry in Retrospect with a New Introduction and Reflections on the Present Conflict by George F. Kennan, Washington D.C. : Carnegie Endowment for International Peace, 1993.

17. Voir Elshtain (Jean Bethke), « Sovereignty, Identity, Sacrifice », Social Research, 58, 1991.

18. Une image véhiculée avec force par Kaplan (Robert D.), Balkan Ghosts: A Journey through History, New York : St. Martin's Press, 1993.

19. Voir Keegan (John), A History of Warfare, Knopf, 1993, pp. 61-76.

20. Cf. Brubaker (Rogers), Nationalism Reframed: Nationhood and the National Question in the New Europe, Cambridge : Cambridge University Press, 1996, pp. 55-76.

21. Čolović (Ivan), Divlja književnost: Etnolingvističko proućavanje paraliterature [La littérature sauvage : étude linguistique de la paralittérature], Belgrade : Nolit, 1984 ; Bordel ratnika : Folklor, politika $i$ rat [Le bordel des soldats : folklore, politique et guerre], Belgrade : Biblioteka XX vek, 1993.

22. Srpski Pokret Obnove [Mouvement serbe du renouveau], le parti de V. Drašković, qui se trouvait dans l'opposition avant de devenir Premier ministre de la Yougoslavie il y a quelques mois.

23. Čolović (Ivan), Divlja književnost (op.cit.), p. 286. 
24. Voir Arnaudov (Mihail), « Naroden epos » [Épopée populaire], Epos, 2 ; Arnaudov (Mihail), ed., Bulgarski narodni pesni [Chants populaires bulgares], Sofia: Hemus, n.d., p. ix; Radost (Ivanova), Epos, Obred, Mit [Épopée, rite, mythe], Sofia : BAN, 1995 ( $2^{\text {nd }}$ éd.), pp. 129-131.

25. Nezavisna Država Hrvatska, État indépendant croate.

26. Sugar (Peter F.), «Ethnicity in Eastern Europe », in Sugar (Peter F.), ed., Ethnic Diversity and Conflict in Eastern Europe, Santa Barbara : ABC-Clio, 1980, p. 432.

27. Pavlowitch (Stevan K.), The Improbable Survivor: Yugoslavia and Its Problems, 1918-1988, Columbus : Ohio State University, 1988, p. 94.

28. Cf. Smith (Anthony D.), Nations and Nationalism in a Global Era, Oxford : Polity Press, 1995, p. 160; Anderson (Benedict), Imagined Communities: Reflections on the Origin and Spread of Nationalism, London : Verso, 1991 (rev. ed.), pp. 10-12.

29. Hughes (Robert), interview à 60 Minutes, CBS, 28/12/97.

30. Voir Steiner (George), In Bluebeard's Castle : Some Notes towards the Redefinition of Culture, New Haven : Yale University Press, 1971, pp. 23-24.

31. Cf. Brubaker (Rogers), Citizenship and Nationhood in France and Germany, Cambridge : Harvard University Press, 1992, p. 72.

32. Voir Plumb (J. H.), The Death of the Past, Boston: Houghton Mifflin, 1969 ; Bridenbaugh (Carl), « The Great Mutation », American Historical Review, 68 (2), 1963.

33. Cf. Čolović (Ivan), Divlja književnost (op.cit.); Sutton (David), Images of History: The Past and Present on a Greek Island, Ph.D. diss., University of Chicago, 1995.

34. Voir Smith (Anthony D.), op.cit., pp. 34-35.

35. Ibid., p. 34.

\section{AUTEUR}

\section{IVELIN SARDAMOV}

I. Sardamov est professeur à l'université américaine de Bulgarie. Ce texte a été présenté à la 4 ème convention annuelle de l'Association for the Study of Nationalities (New York, 15-17/04/99). 